\title{
A Genetic Algorithm-based Framework for Soft Handoff Optimization in Wireless Networks
}

\author{
Daniel E. Asuquo ${ }^{1}$, Samuel A. Robinson ${ }^{1}$ \\ ${ }^{1}$ Department of Computer Science, Faculty of Science, University of Uyo, P.M.B. 1017, 520003, Uyo, Nigeria \\ Correspondence: Daniel E. Asuquo, Department of Computer Science, Faculty of Science, University of Uyo, P. M.B. \\ 1017, 520003, Uyo, Nigeria.
}

Received: June 10, 2016

Accepted: August 6, 2017 Online Published: August 11, 2017

doi:10.11114/set.v5i1.2590

URL: https://doi.org/10.11114/set.v5i1.2590

\begin{abstract}
In this paper, a genetic algorithm (GA)-based approach is used to evaluate the probability of successful handoff in heterogeneous wireless networks (HWNs) so as to increase capacity and network performance. The traditional handoff schemes are prone to ping pong and corner effects and developing an optimized handoff scheme for seamless, faster, and less power consuming handoff decision is challenging. The GA scheme can effectively optimize soft handoff decision by selecting the best fit network for the mobile terminal (MT) considering quality of service (QoS) requirements, network parameters and user's preference in terms of cost of different attachment points for the MT. The robustness and ability to determine global optima for any function using crossover and mutation operations makes GA a promising solution. The developed optimization framework was simulated in Matrix Laboratory (MATLAB) software using MATLAB's optima tool and results show that an optimal MT attachment point is the one with the highest handoff success probability $(P S H)$ value which determines direction for successful handoff in HWN environment. The system maintained a 90\% PSH with 4 channels and more while a 75\% $P S H$ was obtained even at high traffic intensity.
\end{abstract}

Keywords: genetic algorithm, handoff optimization, mobility management, heterogeneous wireless networks

\section{Introduction}

In wireless networks, the ability to effectively manage users' mobility and satisfy desired quality of service (QoS) has been raising much interest among researchers for more than a decade now. The availability of varying wireless technologies creates heterogeneity and provides a variety of new applications (such as multimedia) that ease smooth transition across multiple wireless network interfaces. Future generation communication systems have to provide solutions to the challenges of heterogeneous wireless networks (HWNs) by satisfying mobile users QoS requirements. The 4G systems have mechanisms for providing high data transfer rates, effective user control, and seamless mobility. Many mechanisms for internetworking such as tightly coupled and loosely coupled models that combine different wireless technologies have been proposed (Buddhikot et al., 2003; Salkintzis, 2004; 3GPP, 2006; Verma et al., 2009). However, apart from mobility issues relating to handoff, resource allocation, location management, security and pricing, etc. are also challenging tasks in heterogeneous networks. One of the mobility management components is handoff optimization which regulates how the mobile terminal (MT) changes point of attachment during active communication (Akyildiz et al., 2004). Evolutionary Algorithms (EAs) constitute a promising class of solution techniques to effectively treat large and complex network optimization and design problems having many variables with multiple objectives. In wireless networks, EAs have widespread application in base station placement, channel allocation, and mobility management. Figure 1 shows a diagram of HWN scenario with UMTS/GPRS, WLAN, Wi-Fi, WiMAX, etc. as examples while figure 2 shows a diagram of horizontal and vertical handoffs in overlay networks.

Handoff can be described as the process of changing the communication channel used in the current connection while a call is in progress. This process is extremely important in HWNs when considering the cellular architecture employed so as to maximize spectrum utilization. However, it faces a lot of issues associated with mobility scenario, decision parameters, decision strategies and procedures. Handoff scenario can be horizontal or vertical (Ylianttila et al., 2001; Zhu \& McNair, 2004; Akhila et al., 2012).The former describes the movement of MT from one cell site to another of a homogeneous access network while the later describes a similar movement to a different (heterogeneous) access network. In HWNs, vertical handoff can be initiated for connectivity or convenience sake but such decision may depend 
on a number of influencing parameters which may be network-related, terminal-related, user-related and service-related parameters. The network-related parameters are mainly concerned with bandwidth, latency, Received Signal Strength (RSS), Signal to inference and noise ratio (SINR), cost, security and channel of transmission, etc. The terminal- related parameters are velocity, battery power, location information, etc. User-related parameters deal with user profile and preferences, while service-related parameters deal with service capacities and QoS, etc. Table 1 shows the behaviour of parameters that describe horizontal and vertical handoffs (Abah et al. 2014).

Table 1. Parameters distinguishing vertical and horizontal handoff

\begin{tabular}{lll}
\hline Parameter & Horizontal Handoff & Vertical Handoff \\
\hline Access Network & Does not change & Changes \\
QoS Parameters & Does not change & May change \\
IP Address & Changes & Changes \\
Network Interface & Does not change & Changes \\
Network Connection & Single & Multiple \\
\hline
\end{tabular}

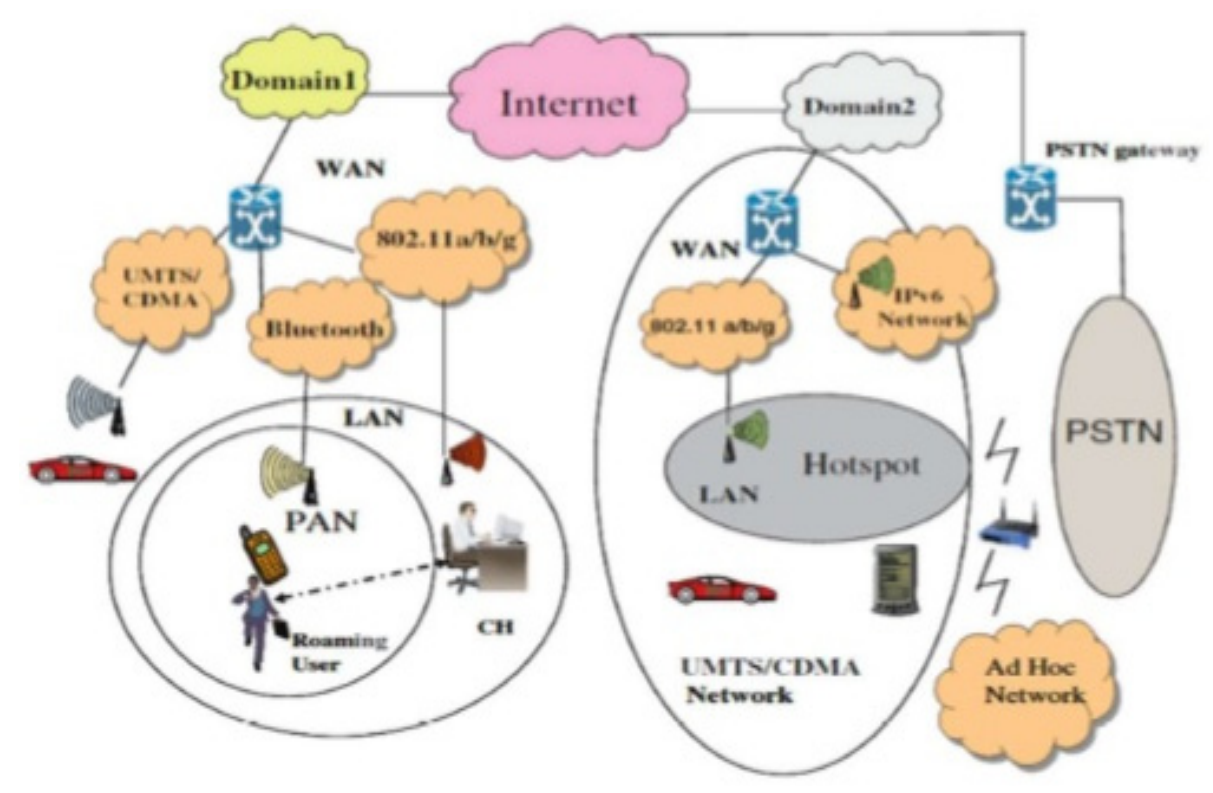

Figure 1. Typical Heterogeneous Wireless Networks

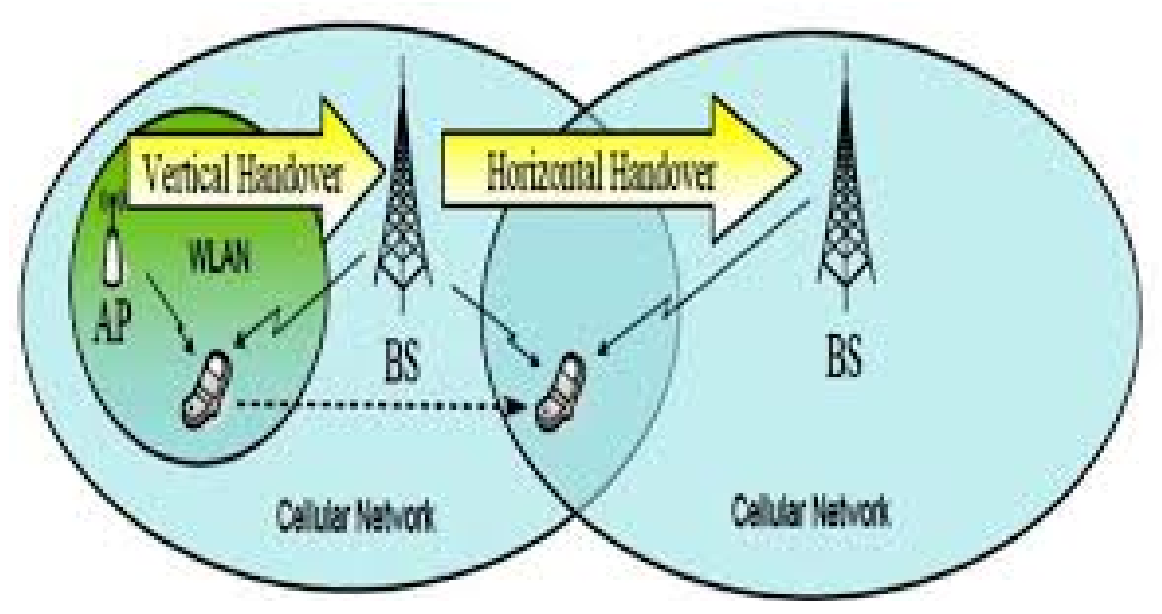

Figure 2. Vertical and Horizontal Handoff scenarios in Wireless Network 
The handoff procedure can be characterized as hard or soft handoff. Hard handoff occurs when the MT breaks communication with the previous point of attachment before making connection with another. It is termed 'break-before-make' handoff because the mobile user should release the old channel in the old cell before a new channel is allocated for it in the new cell. On the other hand, soft handoff termed 'make-before-break' handoff requires that the MT makes a connection with a new point of attachment before breaking from the previous point. In this technique, when the mobile user moves from one cell to another, it can keep the old channel in the first cell until it is assigned a new channel in the new cell so that it guarantee no cut in service or forceful termination. Thus, a conditional decision is made not to/to handoff. This decision is mostly based on the received signal quality from the candidate base stations where the user has simultaneous traffic channel communication with all candidate base stations. Using this parameter, the user will eventually communicate with only one of the base stations. Vertical handoff decision algorithms are helpful in ensuring that the MT is connected to only the best among available networks. The process of vertical handoff is divided into three steps namely, system discovery or handoff initiation, handoff decision, and handoff execution (Lee et al., 2007; Syuhada \& Mahamod, 2008). During system discovery, the MT uses its multiple interfaces to determine what services are available in the identified networks. During handoff decision phase, the MT selects the best network from the available access networks for handoff. During handoff execution phase, connections including user's context information are re-routed from the current network to the best access network in a seamless manner.

Several parameters have been considered for use in the vertical handoff decision algorithms. These include received signal strength which is closely related to service quality and can be measured (Chen et al. 2009), network connection time which refers to the extent that a user remains connected to the current point of connection, available bandwidth which is a calculation of existing data communication resources, expressed in bit per second (Inayat et al., 2005; Alkhayat et al., 2009), power consumption which becomes a critical issue when the battery of MT is low (Syuhada \& Mahamod, 2008; Chandralekha \& Behera, 2011), monetary cost which must be considered in making handover decisions as every network has its own pricing policies (Chen \& Hsut, 2006;Saeed, 2011), security which is considered before selecting one network over another when the integrity of the transmitted data is vital, user preferences since a user's individual liking could lead to the selection of one type of network over the other networks (Lee et al., 2007; Tomar, 2009).

The RSS-based algorithms have received signal strength as the most important criteria. Vertical handoff decision algorithms evaluate the RSS of the current connection against the others to make handover decisions (Alkhayat et al., 2009). The main benefit of this method is that it minimizes handover failures, unnecessary handovers and connection breakdowns as well as handover delay. The bandwidth-based vertical handoff considers the remaining bandwidth, user service requirements, and position of the MT in deciding whether to handover from one access network to another (Lee et al., 2009). If the MT is in the idle state a handover to the preferred access network is performed. It gives good throughput and achieves lower handover latency for delay-sensitive applications. However ping-pong effect may be introduced by these algorithms. The cost function based vertical handoff decision algorithm chooses a combination of network and other factors such as RSS, available bandwidth, service cost, network covering area, security, battery power to estimate the performance of target networks. Accordingly, the handoff decision can then be made. In this method cost of each possible target network is calculated according to priority. This method is useful due to the use of cost function. It reduces the handover blocking probability and increases percentage of user fulfilled requests. In cost function based handover decision algorithm the normalization and weights distribution methods are provided and the network which has the highest weight function is selected as the handover target. It has the benefit of reduced handover decision delay high throughput and low handover blocking rate (Lee et al., 2007). Furthermore, a hybrid algorithm uses various parameters or multiple criteria in the handover decision which relies on computational intelligence. This approach makes vertical handoff decision by applying any computational intelligence technique such as Fuzzy Logic (FL), Fuzzy Multiple Attribute Decision Making (FMADM), Artificial Neural Networks (ANN), Simulating Annealing (SA) or Genetic Algorithm (GA).Bin and Xiaofeng (2012) proposed a speed adaptive policy enabled vertical handoff decision algorithm based on type-2 Fuzzy logic to discover and select best handoff candidate network with maximum throughput for vehicular heterogeneous network. In Chandralekha \& Behera (2010), user preference along with ANN was used to execute handoff for the best offered service among different reachable networks. Generally, computational intelligence based handoff decision algorithms have high implementation complexity. However, they improve user's satisfaction during roaming and have high efficiency. Finally, context-aware approaches employ knowledge of context-awareness of information relating to the MT and the network. This approach considers user information, network and device to guarantee high quality of service and to maintain connectivity for high level user's satisfaction

The existing methods have some drawbacks in that some of them have not considered QoS requirements, network parameters, and the nearest base station congestion while the call is being transferred. The QoS may drop below regulated threshold value and connections are often dropped if handoff requests are not granted at the right time. Also, they do not take into account users' preference and the various attachment options for the mobile user. They achieve low 
throughput and are faced with ambiguous handoff decisions, increased complexity, sometimes causing superfluous handoffs and ping pong effects which are unnecessary in handoff process. So there is a need to develop a new optimized handover technique for improved service delivery in cellular networks. The conventional methods for handoff optimization are not reliable and cost effective. They pose difficulty in implementation and are suitable for static situations, only. In this paper, Genetic Algorithm (GA) is applied for optimization of soft handoff decisions in wireless networks. The use of GA mutation, crossover, selection, and recombination techniques can greatly improve the performance of the system. The parallel and fast convergence nature of GA makes it suitable and scalable for solving optimization problems in a wide range of applications including wireless networks.

The paper is structured as follows. Section 1 presents this introduction. Section 2 reviews related works on handoff optimization techniques, genetic algorithm and its fitness function. Section 3 focuses on the methodology used to achieve low handoff latency, taking into consideration dynamic influencing parameters like interface activation interval of MT, radius of coverage area, distance between base station (BS) and MT for the invocation of the GA optimizer. Also, the framework of the GA-based handoff optimization is presented. Section 4 discusses simulation results while section 5 gives concluding remarks.

\section{Related Works}

Modern wireless network design is challenging due to the dynamic environment under which users' services are provided thereby making parameter optimization a complex task. This dynamic, and often unknown, operating conditions increases wireless networking standards reliance on machine learning and artificial intelligence algorithms (Mehboob et al., 2014). Genetic algorithms (GAs) provide a well-established framework for implementing artificial intelligence tasks such as classification, learning, and optimization. Their versatility makes them remarkably useful in a wide range of application domains including wireless networks. As a meta-heuristic computational method (Hillier \& Lieberman, 2001), GA has been successfully applied in aircraft and telecommunication industry, chip design, computer animation, software creation, and financial markets (Mitchell, 1998). According to Ridley (2004), these algorithms are inspired from biological evolution by imitating and adapting robust procedures used by various biological organisms. In the early days of evolutionary computing, developments took place rather independently from each other (Fogel, 1998), and this led to the emergence of different subareas such as evolutionary programming, evolution strategies, genetic algorithms, and genetic programming (Eiben \& Smith, 2003). The common underlying idea, behind all these techniques is the same such that with a given population of individuals, natural selection (survival of the fittest) is invoked by environmental pressure to grow the fitness of the population. This is a clear optimization process.

Given an objective function to be maximized, one can randomly create a set of candidate solutions and apply the objective function as an abstract fitness measure - the higher the better. Based on this fitness, some of the better candidates are chosen to seed the next generation by applying recombination and mutation to them. Recombination is a binary operator applied to two selected candidates (the so-called parents) and results one or two new candidates (the children). Mutation is a unary operator, applied to one candidate and results in one new candidate. Executing recombination and mutation leads to a set of new candidates (the offspring) that compete - based on their fitness - with the old ones for a place in the next generation. This process can be iterated until a solution is found or a previously set computational limit is reached. In this process, there are two major forces driving progress; 1) selection that acts as a force pushing quality, and 2) variation operators (recombination and mutation) that create the necessary diversity, thereby pushing novelty. Their combined application leads to improving fitness values in consecutive populations showing that evolution is optimizing. Many components of an evolutionary process are stochastic. Variation operators are normally stochastic, since the choice on which pieces of information will be exchanged during recombination, as well as the changes in a candidate solution during mutation, are random. Selection operators can be either deterministic, or stochastic. In the latter case, fitter individuals have a higher chance to be selected than less fit ones, but typically even the weakest individuals have a chance to become a parent or to survive. The general scheme of an evolutionary algorithm can be given as follows:

INITIALIZE population with random individuals (candidate solutions)

EVALUATE (compute fitness of) all individuals

WHILE not TERMINATION CONDITION DO

SELECT parents

RECOMBINE pairs of selected parents

MUTATE the resulting offspring

EVALUATE the offspring

SELECT new population from last population and offspring 
In Wang et al. (1999), a policy-enabled handoff across heterogeneous network environment using cost function was proposed. The idea is to use the cost function for MT's handoff decision and as a measurement of the benefit obtained by handing off to one of these access networks. Available bandwidth, power consumption, and service cost were the considered parameters. Consequently, a cost function-based vertical handoff decision algorithm for multi-services was presented in Zhu \& McNair (2004). The access network with the lowest cost function value becomes the handoff target amongst available networks. However, only available bandwidth and RSS were considered in the decision algorithm. A network selection algorithm that employs analytical hierarchy process (AHP) and grey relational analysis (GRA) as multiple attribute decision making (MADM) techniques was presented in Songand Jamalipour (2005) with a number of parameters. Multiplicative Exponent Weighting (MEW), Simple Additive Weighting (SAW), and Technique for Order Preference by Similarity to Ideal Solution (TOPSIS) are also classical applicable MADM methods (Savitha \& Chandrasekar, 2010; Asuquo \& Onuodu, 2016).

Stoyanova\& Mahonen(2007) used MEW, SAW, and TOPOSIS algorithms with a number of attributes in their vertical handoff decision for four traffic classes. The traffic classes are conversational, streaming, interactive and background. Results from simulation showed that MEW, SAW and TOPSIS provide similar performance to all traffic classes. Savitha \& Chandrasekar (2011) used GRA to rank available networks and select the one with the highest ranking for handoff while Radhika \& Venugopal (2013) used AHP to decompose the network selection problem into several sub-problems, assigning weighted score to sub-problems in the AHP process. In Nikhil \& Kiran (2012), a dynamic decision model for vertical handoff was proposed. The model comprises a three phase approach namely, priority phase, normal phase and decision phase. Priorities are assigned to candidate networks in the first phase while system's information and user preferences are recorded in the second phase. Later, a cost function for each candidate network is computed and finally, in the last phase, a score function for each candidate network is computed where the network with the highest score (highest quality and lowest cost) is selected as "best network" for handoff and all ongoing transmissions are connected to it. This method is able to maximize user's satisfaction.

In Shen \& Zeng (2006), a novel handoff decision strategy aimed at reducing unnecessary handoffs was considered in order to meet users' needs. The time-adaptive strategy makes right and timely handoff decisions by adjusting MT's interface activating interval based on user's movement and prevailing network conditions. Radhika \& Venugopal (2011) presented a decision algorithm for handoff using Game Theory approach. The algorithm enables mobile users choose the optimal network based on Bayesian Nash-equilibrium point which maximizes offered service quality with minimum delay and cost. Artificial intelligence approaches such as genetic algorithm, fuzzy logic, and neural network can be applied to choose when and over which network to hand over among different available access networks. These methods can be combined with multiple criteria or attribute decision making methods in order to develop advanced decision algorithms for both non-real-time and real-time applications. Dhand \& Dhillon (2013) proposed a handoff optimization scheme in wireless and mobile networks using fuzzy logic controller. They argued that traditional handoff algorithms using fixed parameter values can only perform creditably in specific environments but a fuzzy controlled handoff scheme adds more capabilities to provide adaptation to dynamic environments. In Nasser et al. (2006), artificial neural network was used to control and manage handoffs across heterogeneous wireless networks. The method was capable of selecting the best existing network that satisfies set users' preferences as the MTs attempt vertical handoff. In Wei et al. (2006), an adaptive quality of service handoff priority scheme for mobile multimedia networks was proposed. Ylianttila et al. (2002) proposed a hybrid approach to vertical handoff decision based on fuzzy logic and neural networks. Although fuzzy logic and neural network approaches are good for pattern classification due to their non-linearity and generalization capabilities, using knowledge of context information of the MT and available networks to take intelligent and improved handoff decision offers promising solutions. Thus, this paper considers the use of GA to optimize soft handoff decision in wireless networks with dynamic channel configurations. Often times, GAs avoid local optima with a high probability using the mutation and crossover operators, thereby providing satisfactory performance in changing network conditions.

\subsection{Overview on Genetic Algorithm}

Genetic algorithms are considered part of optimization techniques that mimics the process of natural evolution. The method solves both constrained and unconstrained optimization problems based on the principle of natural selection by repeatedly modifying a population of individual solutions such that at each step, it selects individuals at random from the current population to be parents and uses them to produce the children for the next generation. This is done in a loop towards an optimal solution. It can be said however that most methods of genetic algorithm have these elements in common (Weise, 2009): populations of chromosome; selection according to fitness; crossover to produce new offspring; and random mutation of new offspring. The chromosomes of genetic algorithm usually take the form of bit strings. The pseudocode of a simple genetic algorithm is presented in figure 3 with selection, crossover, and mutation operators. 


\author{
Function SGA () \\ \{ \\ initialize population; \\ calculate fitness function; \\ whileTerminationCriteriaNotSatisfied \\ \{ \\ select parents for reproduction; \\ perform recombination and mutation; \\ evaluate population; \\ \}
}

Figure 3. Pseudocode of a simple GA

The selection process determines the solutions that should be preserved for reproduction while allowing others to die out. Thus, the selection operator simply selects the best solution and discards the rest while keeping the population size constant. The crossover operator randomly chooses a locus between two chromosomes to create two offspring, thereby mimicking biological recombination between two single chromosome (haploid) organisms (Melanie, 1999). The mutation operator randomly flips some of the bits in a chromosome. A fitness function is used for identification of good solution where the fitness value quantifies the optimality of a solution. Thus, the value assigned to each solution depends on how close the solution is to the optimal solution of the problem. Commonly used methods to implement selection are roulette wheel selection method, linear ranking method, and tournament selection method. The theory of roulette wheel selection developed by John (1992) assumes that the chromosomes are selected based on their probabilities that are proportional to their fitness value. The probability of selecting an individual $a_{j}$ is represented as:

$$
p s\left(a_{i}\right)=f\left(a_{i}\right) / \sum_{i=1}^{n} f\left(a_{j}\right) ; j=1,2, \ldots, n
$$

Where $n$ is the size of population, $f\left(a_{i}\right)$ is the fitness value of the individual $a_{j}$. The linear ranking selection in GA was introduced to eliminate the disadvantages of proportionate selection. In this method, individuals are first sorted according to their fitness value and then ranks are assigned to them. The best individual gets rank ' $N$ ' and the worst gets rank ' 1 '. The selection probability is then assigned linearly to the individuals according to their ranks as follows:

$$
P_{i}=\frac{1}{N}\left(n^{-}+\left(n^{+}-n^{-}\right) \frac{i-1}{N-1}\right) ; i=1, \ldots N
$$

where $P_{i}$ is the selection probability of the $i_{t h}$ individual, $n^{-} / N_{N}$ is the probability of selecting the worst individual, and $\mathrm{n}^{+} / \mathrm{N}$ is the probability of selecting the best individual. In tournament selection, individuals are chosen at random from

the population where several tournaments are played among a few of them and the winner at each tournament level is selected for the next generation.

The performance of the algorithm is enhanced by finding a chromosome that stores specific information about the problem as well as a possible solution to the problem setting. The objective or evaluation function is what provides the mechanism for evaluating the status of each chromosome by creating a link between the genetic algorithm and the system. It takes a chromosome (potential solution) as input and produces a number (objective value) which is a measure of the chromosome's performance indicating how well that chromosome solves the problem. To maintain uniformity, a fitness function is used to map the objective value to a fitness value proportional to the utility of the individual which that chromosome represents. Although there are a number of techniques used for this mapping the two most commonly used are windowing and linear normalization. During reproductive phase, individuals are selected from the present population and recombined to produce offspring that will constitute the next generation.

\title{
2.2 Handoff Decision in Wireless Networks
}

Handoff can be initiated for reasons that deal with the quality of service experienced by the MT such as channel interference, changes in user behaviour, lack of capacity in current cell, and need to avoid call termination. During handoff, packet loss is likely to occur and one of the goals of mobility management is to minimize packet loss. It also ensures provision of seamless handoff, where upper layers (e.g. applications) experience minimal disruption. The various techniques for controlling handoff decision can be classified into mobile-initiated, network-initiated, mobile-controlled, network-controlled, mobile-assisted, and network-assisted handoff (Wang et al., 1999; Shengdong \& 
Meng, 2009). In mobile-initiated handoff, the mobile node makes the initial decision to handoff while the network makes the decision to handoff in network-initiated handoff. In mobile-controlled handoff, the mobile node has primary control over the handoff process while the network has primary control over the handoff process in network-controlled handoff. Finally, in mobile-assisted handoff, information and measurement from the mobile node are used by the core network to decide on the execution of a handoff while network-assisted handoff entails that the core network provides information that can be used by the mobile node in a handoff decision.

In wireless communication networks, basic handoff algorithms use traditional handoff decision schemes where target network is compared with current network based on received signal strength indication(RSSI)(Kaur \& Sood, 2013). The disadvantage of choosing RSSI is that when the MT moves near the cell boundary of two BSs, the received signal strengths from both BSs will be very close. Moreover, the strength of signal will be more susceptible to shadowing effect, capable of causing unnecessary handoff between the two BSs back and forth constantly. This problem is called ping-pong effect. The decision is made based on the RSSI at the edge of the two networks coverage area using the ideal coverage concept or adaptive handoff decision method. The idea is to perform evaluation on the candidate networks using utility function, utility index, and utility ratio. The utility function provides a value indicating QoS of the wireless networks. The utility function of network $j$ from the view of mobile user is composed of several normalized factors $f_{i, j}$ that are multiplied with their weights of importance $w_{i}$ as given in equation (3).

$$
\text { Utility }_{j}=\sum_{i} w_{i} \times f_{i, j}
$$

where $w_{i}$ is the weight of factor $i$ and $f_{i, j}$ is factor $i$ of network $j$.

The weight of factors may vary in different applications. As a result there is need for normalization of factors. This is done by defining acceptable maximum and minimum values for each factor. The normalized factor is zero if the real factor value $R_{i, j}$, is less than or equal to minimum and is one for the reverse. The mapping of real factor value to normalized factor for network $j$ is given in equation (4) as:

$$
f_{i, j}=\left\{\begin{array}{c}
0, \text { if } R_{i, j} \leq L B_{i} \\
1, \text { if } R_{i, j}>U B_{i} \\
\frac{R_{i, j}-L B_{i}}{U B_{i}-L B_{i}}, \text { otherwise }
\end{array}\right.
$$

where $L B_{i}$ and $U B_{i}$ are lower bound and upper bound of the $\mathrm{i}^{\text {th }}$ factor, respectively.

The utility ratior, is the ratio between the two networks, i.e. utility of the target vs. utility of the current network. It is evaluated in equation (5) as:

$$
r=U_{-} \text {target } / U_{-} \text {current }
$$

If the target utility is higher, the system checks if that target network is consistently higher by calculating the stability $\mathrm{T}_{\text {stability }}$ as in equation (6):

$$
T_{\text {stability }}=l_{\text {handoff }}+\frac{l_{\text {handoff }}}{r-1}
$$

The stability is measured in intervals, determined by the last utility evaluation result. Hence, the interval between the $m_{t h}$ evaluation and the $m+1_{t h}$ evaluation is given in equation (7) as:

$$
T_{\text {interval }}=\frac{l_{\text {handoff }}}{N}+\frac{l_{\text {handoff }}}{N \times\left(r_{m}-1\right)}
$$

where $l_{\text {handoff }}$ is the last handoff while $r_{m}-1$ is the utility obtained in the $\mathrm{m}-1^{\text {th }}$ evaluation.

A MT may handoff as soon as possible if the utility ratio increases and may not want to handoff if the utility ratio decreases. In this model, an evaluation of the system shows that they are more sensitive than static decision algorithms even when the initial utility ratio is high. Handoff decision in wireless networks is a challenging task and tradition handoff schemes are not sufficient to deal with seamless mobility demand in the presence of context-aware services. They increase complexity and ambiguity in handoff decision as user's velocity increases leading to increase call drop rate. Hence, the need for optimization approaches.

\section{Methodology}

The optimized handoff algorithm is expected to make faster handoff decision so as to reduce latency and service degradation. The framework for optimizing soft handoff decision is based on the use of GA technique, as presented in figure 4 while figure 5 presents the flowchart showing required GA operations and terminating criteria for the framework. Furthermore, a fitness function is developed to ensure high handoff success probability which can result to timely network discovery and handoff decision. 


\subsection{GA-based Handoff Performance Optimization}

The major components of the framework comprise the GA-based optimizer, Database Server, Mobile Terminal, and Wireless Access Networks. The proposed GA-based framework takes into consideration several wireless networks such as WiMAX, WLAN, 3G-UMTS and 4G-LTE. The major focus is on optimization of soft handoff such that the MT will have the ability to scan and find the best available access networks on time. In optimizing handoff decision to select the best available network, the GA technique takes these three major steps:

Step 1: The algorithm creates a random initial population of wireless mobile nodes.

Step 2: Asequence of new population of mobile nodes is created from individuals in the current generation by iteratively performing the following operations:

- Evaluating the fitness value of candidate solutions in the current population.

- Selecting better solutions called parents based on their fitness.

- Choosing some individuals with lower fitness values in the current population as elite individuals and pass them to the next population as children.

- Performing crossover by combining the vector entries of two parents to form potentially better solutions (offspring) for the next generation. Crossover is controlled by the crossover probability, pc which is typically in the range $0.7-0.95$.

- Applying random changes to one or more vector entries of an individual parent (mutation) to form children. Mutation, which adds to the diversity of a population, is typically performed with a low probability, pm in the range $0.01-0.2$.

- Replacing the current population with the children created by selection, crossover, and mutation to form the next generation.

Step 3: The algorithm stops once a stopping criterionis established, then the three phases of handoff (network discovery, handoff decision, and handoff execution) become initialized. Otherwise, fitness value evaluation of candidate solutions is revisited.

During handoff discovery phase, the framework activates the wireless interfaces on the MT within an interval, scanning for available networks that overlap with the current network. The activation uses an adaptive discovery scheme adopted by the MT to ensure a potential wireless network is ready for handoff, based on the list stored in the database containing $R_{\max }, R_{\min }$, and the position of the base station. The interval for activation is evaluated using equation (8) as:

$$
T_{\text {interval }}=\left(T_{\max }-T_{\min }\right) \times \frac{R_{\text {current }}-R_{\min }}{R_{\max }-R_{\min }}+T_{\min }
$$

where, $T_{\text {interval }}$ is the interface activating interval, $T_{\max }$ is the upper bound of $\mathrm{T}_{\text {interval }}, T_{\min }$ is the lower bound of $\mathrm{T}_{\text {interval }}, R_{\max }$ is the radius of the ideal coverage, $R_{\min }$ is the radius of the minimum coverage, $R_{\text {current }}$ is the distance between base station and MT.

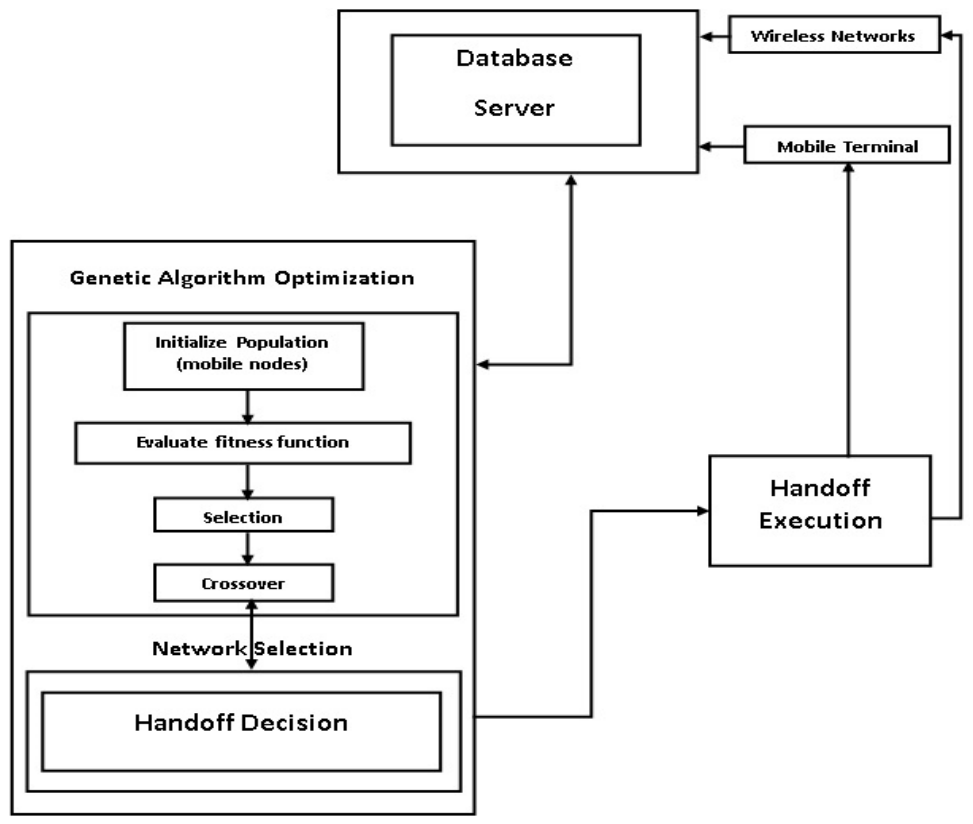

Figure 4. GA-based Framework for Soft Handoff Decision 


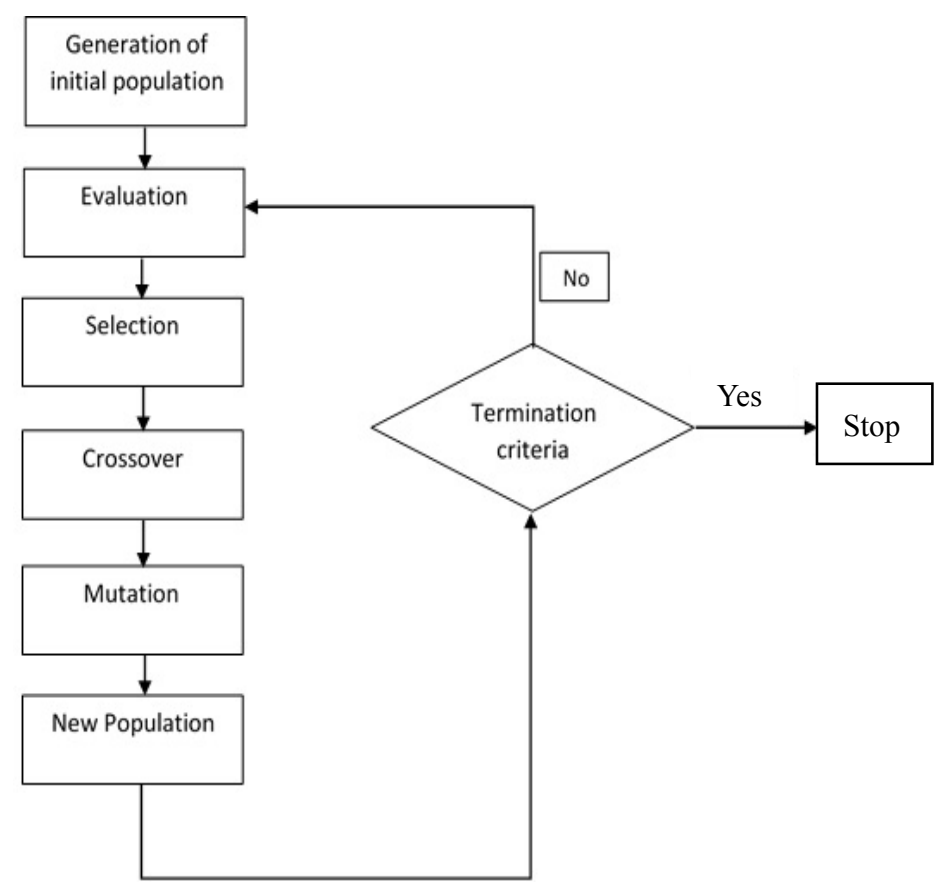

Figure 5. Flowchart of the GA-based Framework

Once the network has been discovered, the framework invokes the GA optimizer to make a decision to handoff. The model in equation (9) evaluates the probability of successful handoff, $P(\mathrm{SH})$ at any cell as:

$$
\begin{gathered}
P(S H)= \\
1-\left[\frac{\frac{k p^{1+n c}}{n c !}\left(d_{r} t\right)^{n}}{n ! \sum_{i=0}^{n c}\left(\frac{\rho^{i}}{i !}\right)} e^{-d_{r} t}\right]-\left[\frac{\rho^{n c} / n c !}{\sum_{i=0}^{n c}\left(\rho^{i} / i !\right)}+\frac{\left(d_{r} t\right)^{n}}{n !} e^{-d_{r} t}+k \rho\right]+\frac{k p^{1+n c} / n c !}{\sum_{i=0}^{n c}\left(\rho^{i} / i !\right)}+\frac{k \rho\left(d_{r} t\right)^{n}}{n !} e^{-d_{r} t}+ \\
\frac{\frac{\rho^{n c}}{n c !}\left(d_{r} t\right)^{n}}{n ! \sum_{i=0}^{n c}\left(\rho^{i} / i !\right)} e^{-d_{r} t}
\end{gathered}
$$

The parameters considered are probability of blocked call $\mathrm{P}(\mathrm{B})$, probability of call congestion $\mathrm{P}(\mathrm{C})$, and probability of dropped call $\mathrm{P}(\mathrm{D})$. These probabilities are evaluated in equations (10) $-(12)$, respectively, where $\rho=\lambda \times t$.

$$
\begin{gathered}
P(B)=\frac{\frac{\rho^{n c}}{n c !}}{\sum_{i=1}^{n c} \frac{\rho^{i}}{i !}} \\
P(C)=k \cdot \rho \\
P(D)=\frac{\left(d_{r} t\right)^{n}}{n !} e^{-d_{r} t}
\end{gathered}
$$

$n c$ is the number of channels, $\rho$ is the traffic intensity, $k$ is a constant, $d_{r}$ is the dropped calls rate, $t$ is the call duration, $n$ is the number of dropped calls, and $\lambda$ is the number of carried connections per unit time.

\section{Results and Discussion}

The focus of this paper is on the decision phase where the genetic algorithm is applied to help MT find the optimal target network to connect with and on time. Deploying GA is aimed at maximizing the probability of successful handoff. A database on the MT is used to store information obtained from the wireless network cell site. The algorithm takes $P(S H)$ as its fitness function, and performs evaluation with the parameter values obtained from available cell sites of appropriate wireless networks. The parameters include number of channels, call duration, number of dropped calls, dropped calls rate, and traffic intensity. The constant $k$ in the model is obtained from previously evaluated probabilities. The solution to the problem is the parameter values with the highest $P(\mathrm{SH})$ fitness. Randomly generated parameter values were used in this paper and the results are presented in figures 6-10.

Figure 6 shows a plot of $\mathrm{P}(\mathrm{SH})$ vs. dropped call rate while figure 7 is a graph of $\mathrm{P}(\mathrm{SH})$ vs. number of channels. Results from figure 6 indicate that although success probability of handoff requests decreases with increase in drop call rate, the 
system can still assure seamless handoff to $67 \%$ of roaming users. Figure 7 shows that increasing the number of channels for communication in the network enable more handoff requests to be accepted as handoff success probability increases. Consequently, the algorithm helps the system maintain a $90 \%$ constant handoff success probability when channels availability rises from 5 and above. In figure 8, where a plot of $\mathrm{P}(\mathrm{SH})$ vs. traffic intensity is presented, success probability of handoff requests decreases as traffic intensity increase. The GA still optimized soft handoff decision by ensuring a success probability of $75 \%$ even at high traffic intensity thereby ensuring drastic handoff latency reduction. Figure 9 shows a graph of best individual values vs. the parameters using GA for optimization. As expected the current best individual should have the ability to sustain some connection or traffic intensity and have a reasonable number of channels. Furthermore, the optimal system should have an almost non-existent dropped call rate and be able to permit long call durations. The base station selected is expected to have the closest value to the global optima of the handoff probability model. Figure 10 shows the plot of fitness value in each generation vs. the iteration. This graph indicates that the optimum of the function was reached after the 70-80 generation iterations.

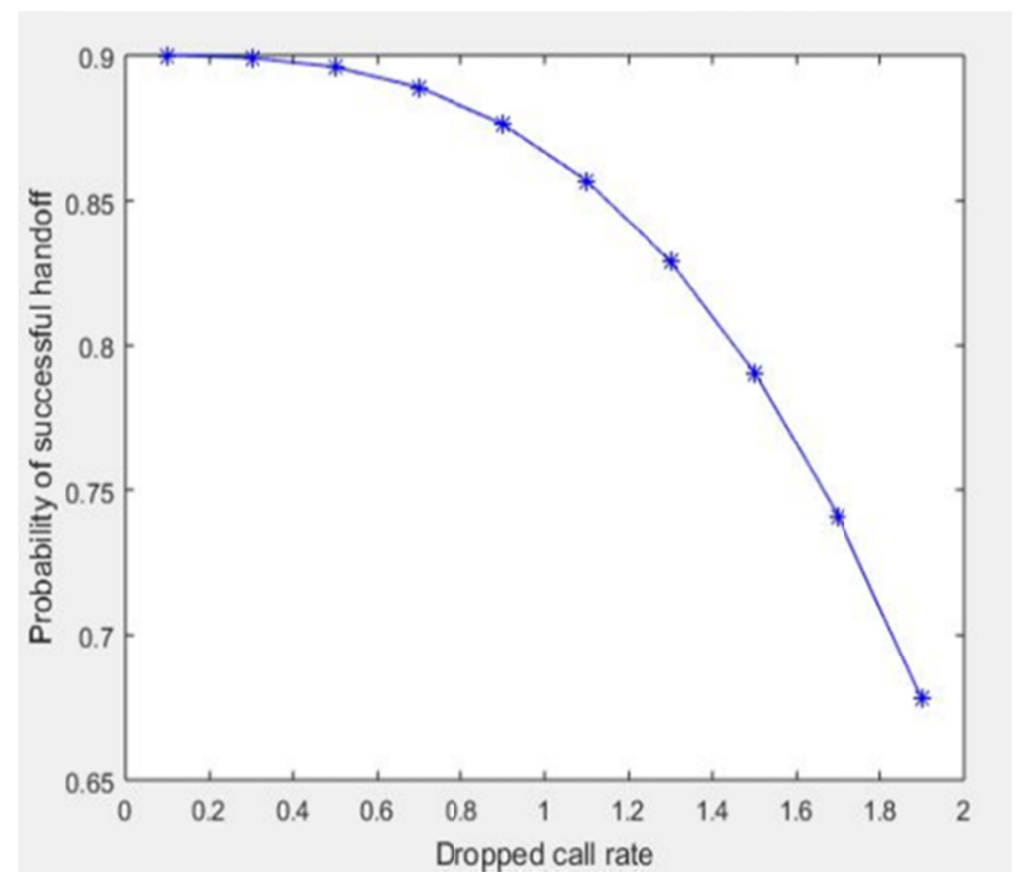

Figure 6. $\mathrm{P}(\mathrm{SH})$ against Dropped call rate

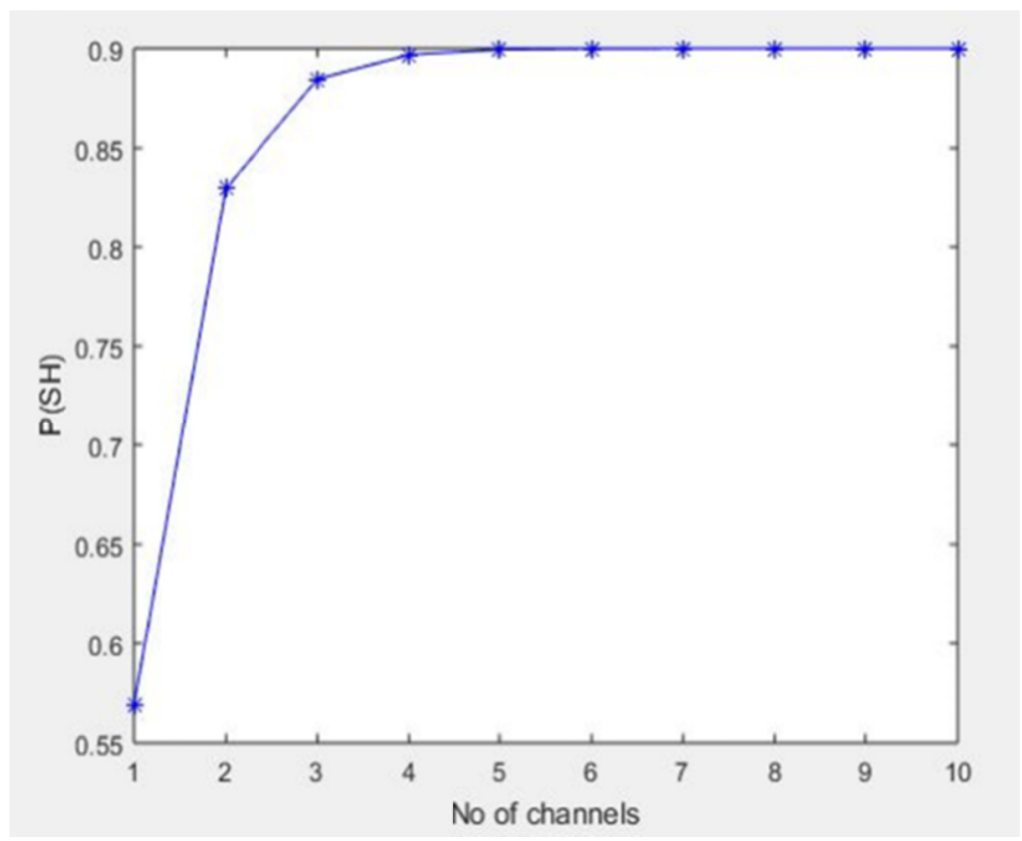

Figure 7. P(SH) against Number of channels 


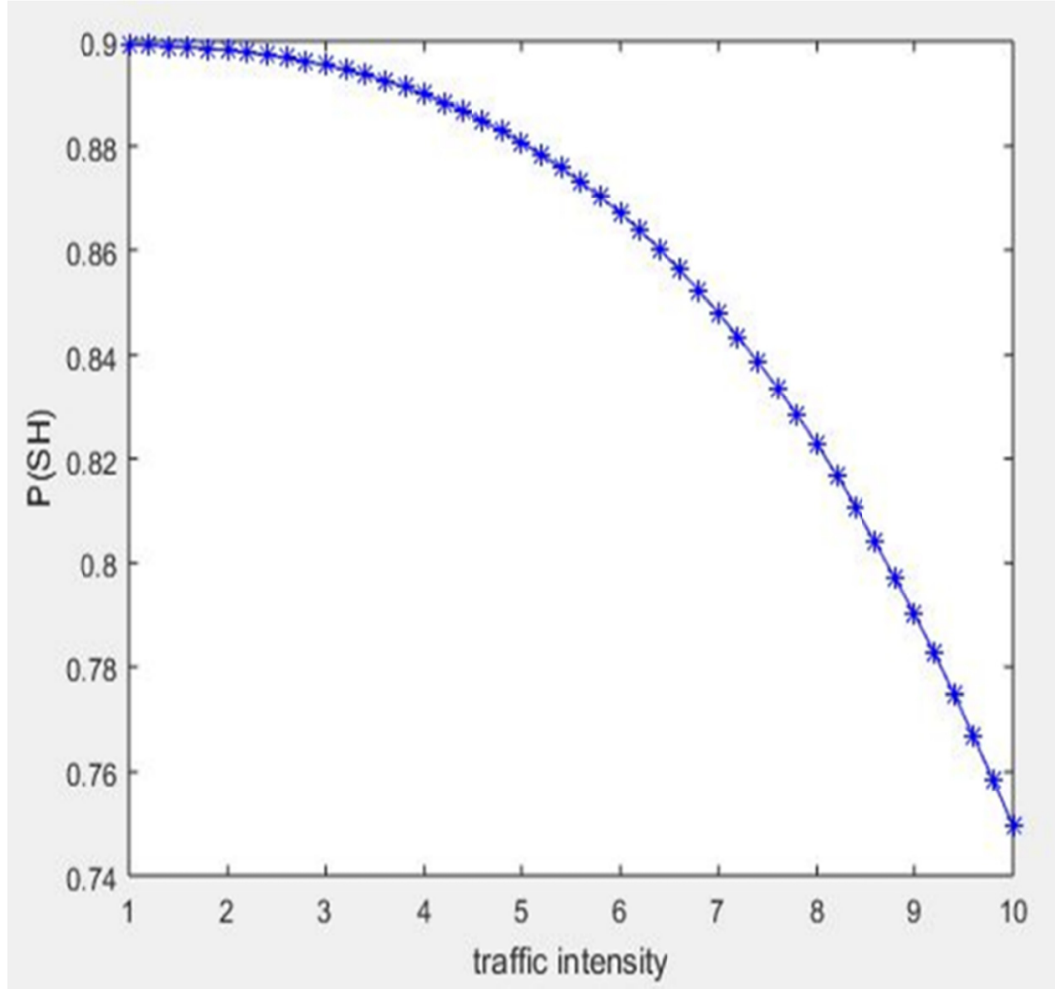

Figure 8. P(SH) against Traffic Intensity

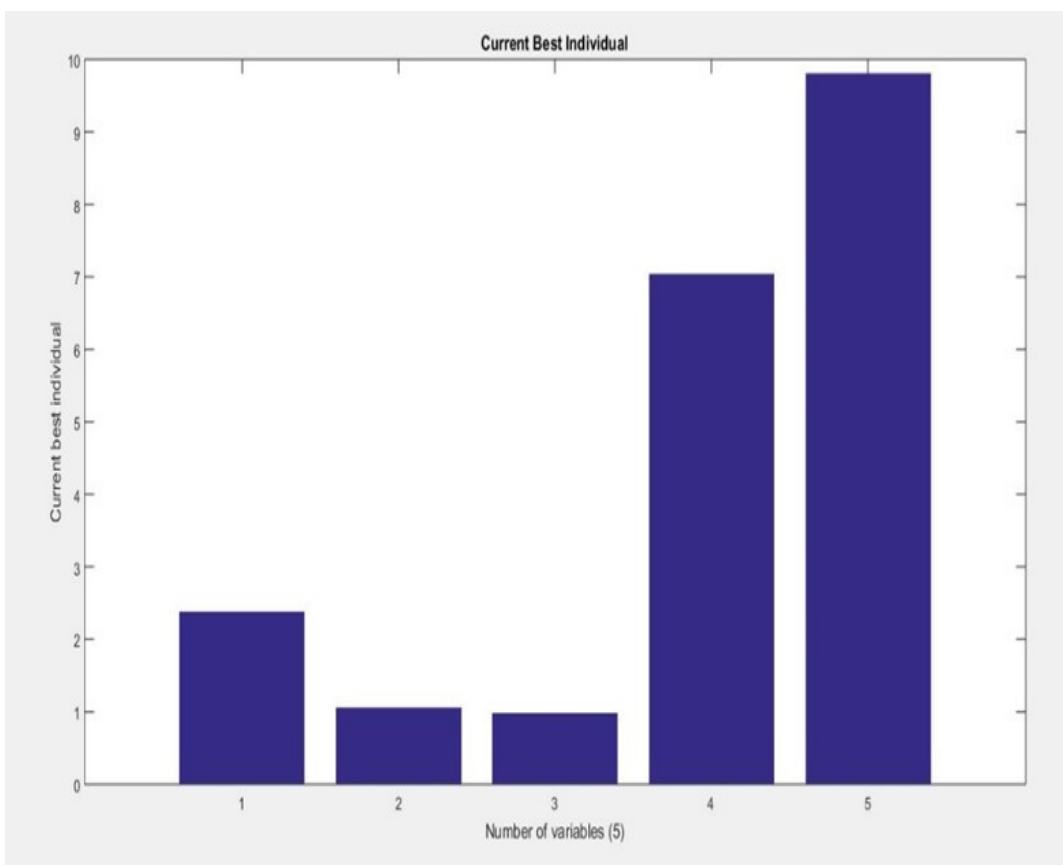

Figure 9. Parameters vs. Number of Variables for Best Individual 


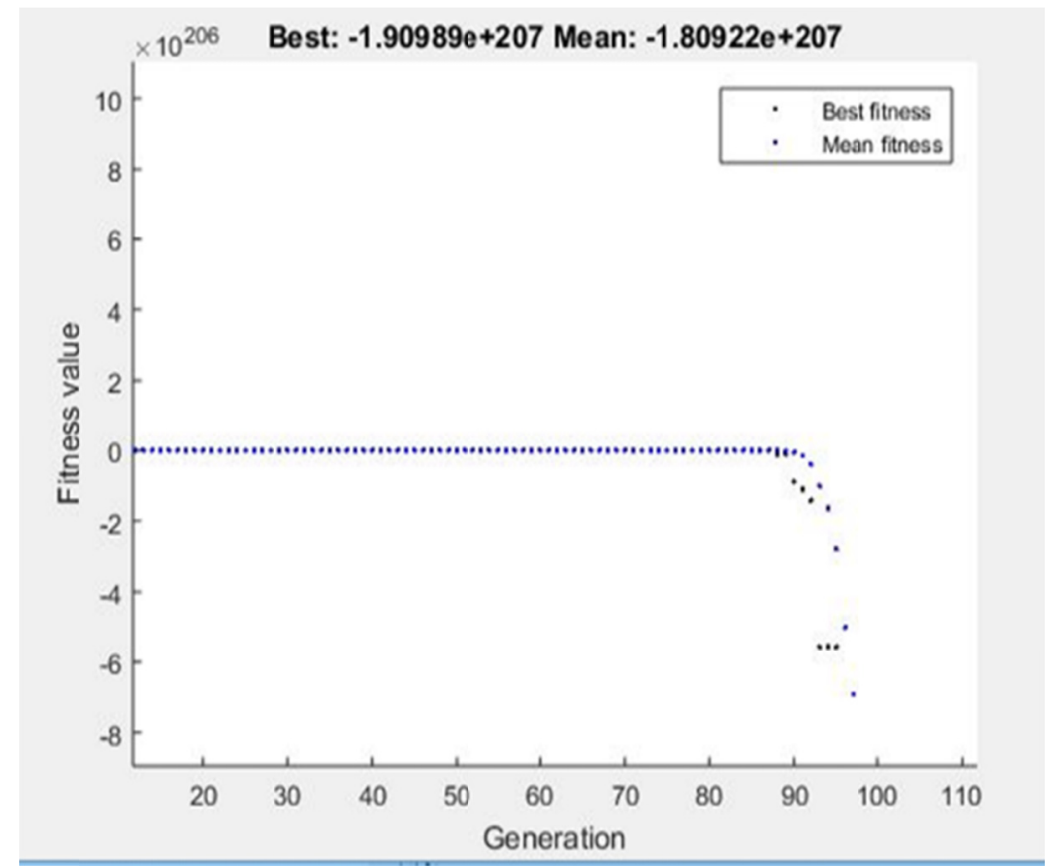

Figure 10. Fitness value plot for each generation vs. the iteration

\section{Conclusion}

Handoffs are extremely important in heterogeneous networks because of the cellular architecture deployed to maximize spectrum utilization. The soft handoff framework in this paper considers important handoff parameters such as number of channels, traffic intensity, average call duration, number of dropped calls and dropped calls rate. To really establish relationships between model parameters, a simulation of the model was carried out to aid prediction after a careful study of the system behavior influenced by the impact of these parameters. This process reveals that with high traffic intensity at minimum of two channels, the system could achieve about $75 \%$ and $83 \%$ handoff success rate, respectively indicating that a telecommunication system can be planned to achieve desired maximum handoff success rate. In conclusion, the GA scheme is simple, efficient, generic, suitable for soft vertical handoff decision and can be adapted to any other wireless network types after obtaining the required simulation parameters. The method can satisfy the QoS requirements of both users and network operators. Future works will consider the use of distributed evolutionary algorithm in a distributed network environment to maintain population diversity, further improve system availability and provide more satisfactory results within reasonable time thereby avoiding local optima and facilitating multi-objective search.

\section{References}

3GPP TR 23.234 v7.1.0. 3GPP System to WLAN Internetworking: System Description (Release 7), March 2006, http://www.3gpp.org/specs/specs.html.

Abah, U. C., Aibinu, A. M., Onwuka, E. N., \& Onumanyi, A. J. (2014). An Overview of Decision Techniques for Vertical Handoff across Wireless Heterogeneous Networks, British Journal of Applied Science and Technology, 4(23), 3322-3331. https://doi.org/10.9734/BJAST/2014/11396

Akhila, S., Jayanthi, K., Murthy, A. R., \& Shankar, S. K. (2012). An overview on decision techniques for vertical handoffs across wireless heterogeneous networks. International Journal of Scientific and Engineering Research, $3(1), 34-56$.

Akyildiz, I. F., Xie, J., \& Mohanty, S. (2004). A Survey of Mobility Management in Next-Generation all IP-based Wireless Systems. IEEE Wireless Communications, 11(4), 16-28. https://doi.org/10.1109/MWC.2004.1325888

Alkhayat, I., Kumar, A., \& Hariri, S. (2009). End-to-End Mobility Solution for Vertical Handoff between Heterogeneous Wireless Networks, $7^{\text {th }}$ IEEE/ACS International Conference on Computer Systems and Applications, 750-755.

Asuquo, D. E., \& Onuodu, F. E. (2016). A Fuzzy AHP Model for Selection of University Academic Staff, International Journal of Computer Applications, 141(1), 19-26.

Bin, M., \& Xiao, F. L. (2012). Speed-adaptive vertical handoff algorithm based on fuzzy logic in vehicular heterogeneous networks. In Proceedings of the IEEE 9th International Conference on Fuzzy Systems and Knowledge Discovery (FSKD), 144-233. 
Buddhikot, M., Chandramenon, G., Han, S., Lee, Y. W., Miller, S., \& Salgarellim, L. (2003). Integration of 802.11 and Third -Generation Wireless Data Networks, IEEE INFOCOM '03, San Francisco, USA, 503-512.

Chandralekha, \& Behera, K. B. (2010).Minimization of number of handoff using genetic algorithm in heterogeneous wireless network. International Journal of Latest Trends in Computing, 1(2), 24-30.

Chandralekha, \& Behera, P. K. (2011). Optimization of Vertical Handoff Performance Parameters in Heterogeneous Wireless Networks, International Journal of Modern Engineering Research, 1(2), 597-601.

Chen, H. P., \& Hsut, W. P. (2006). Adapted Queuing Priority Algorithm for Soft Handoff in Mobile Communication Networks, IEEE. https://doi.org/10.1109/ISCIT.2006.339831

Chen, Y. C., Hsia, J. H., \& Liao, Y. J. (2009). Advanced Seamless Vertical Handoff Architecture for WiMAX and WiFi Heterogeneous Networks with QoS Guarantees, Computer Communications, 32(2), 281-293. https://doi.org/10.1016/j.comcom.2008.10.014

Dhand, P., \& Dhillon, P. (2013).Handoff Optimization for Wireless and Mobile Networks using Fuzzy Logic, International Journal of Computer Applications, 63(14), 31- 35. https://doi.org/10.5120/10535-5527

Eiben, A. E., \& Smith, J. E. (2003). Introduction to Evolutionary Computing, New York: Springer-Verlag. https://doi.org/10.1007/978-3-662-05094-1

Fogel, D. B. (ed.). Evolutionary Computation: The Fossile Record, IEEE Press, 1998. https://doi.org/10.1109/9780470544600

Hillier, F., \& Lieberman, G. (2001). Introduction to operations research, New York: McGraw Hill.

Inayat, R., Aibara, R., \& Nishimura, K. (2005). Handoff Management for Mobile Devices in Hybrid Wireless Data Networks, IEEE Journal of Communications and Networks, 7(1), 76-86. https://doi.org/10.1109/JCN.2005.6387759

John, H. (1992).Adaptation in natural and artificial systems. USA: MITCognet.

Kaur, R., \& Sood, R. (2013).Comprehensive study of Handoff Strategy: A Survey. International Journal of Engineering and Computer Science, 2(12), 3576-3579.

Lee, S., Sriram, K., Kim, K., Kim, Y. H., \& Golmie, N. (2009). Vertical Handoff Decision Algorithms for Providing Optimized Performance in Heterogeneous Wireless Networks, IEEE Transactionson Vehicular Technology, 58(2), 865-881.https://doi.org/10.1109/TVT.2008.925301

Lee, W., Kim, E., Kim, J., Lee, I., \& Lee, C. (2007). Movement-Aware Vertical Handoff of WLAN and Mobile WiMAX for Seamless Ubiquitous Access, IEEE Transactions on Consumer Electronics, 53(4), 1268-1275. https://doi.org/10.1109/TCE.2007.4429211

Mehboob, U., Qadir, J., Ali, S., \& Vasilakos, A. (2014). Genetic Algorithms in Wireless Networking: Techniques, Applications and Issues, Journal of Soft Computing, 20(6), 2467-2501. https://doi.org/10.1007/s00500-016-2070-9

Melanie, M. (1999). An Introduction to Genetic Algorithms. Massachusetts: Fifth printing.

Mitchell, M. (1998).An introduction to genetic algorithms. MIT press.

Nasser, N., Hasswa, A., \& Hassanein, H. (2006). Handoffs in Fourth Generation Heterogeneous Networks. IEEE Communications Magazine, 44(10), 96-103. https://doi.org/10.1109/MCOM.2006.1710420

Nikhil, P., \& Kiran, P. (2012). Quality Dependent Vertical Handover Decision Algorithm for Fourth Generation (4G) Heterogeneous Wireless Networks. IJCEM International Journal of Computational Engineering \& Management, $15(2)$.

Radhika, K., \& Venugopal, R. (2011). Vertical Handoff Decision using Game Theory Approach for Multi-mode Mobile Terminals in Next Generation Wireless Networks, International Journal of Computer Applications, 36(11), 31-37.

Ridley, M. (2004). Evolution (3rd ed.), Blackwell.

Saeed, R. A. (2011). WiFi/WiMAX Seamless Convergence with Adaptive Vertical Handover for Continuity of Internet Access, Advances in Internet of Things, 1, 32-37. https://doi.org/10.4236/ait.2011.12005

Salkintzis, A. K. (2004). Internetworking Techniques and Architectures for WLAN/3G Integration Toward 4G Mobile Data Networks, IEEE Wireless Communications Magazine, 11(3), 50-61. https://doi.org/10.1109/MWC.2004.1308950

Savitha, K., \& Chandrasekar, C. (2010). An Overview of Vertical Handoff Decision Based On MADM For Heterogeneous Wireless Network, Journal of Computer Applications, 3(3), 12-15. 
Savitha, K., \& Chandrasekar, C. (2011). Grey Relation Analysis for Vertical Handover Decision Schemes in Heterogeneous Wireless Networks, European Journal of Scientific Research, 54(4), 560-568.

Shen, W., \& Zeng, Q. A. (2006). A Novel Decision Strategy of Vertical Handoff in Overlay Wireless Networks Fifth IEEE International Symposium on Network Computing and Applications, 227-230.

Shengdong, X., \& Meng, W. (2009).Vertical handoff algorithm in heterogeneous networks to maximize system benefit, Journal of Systems Engineering and Electronics, 20(5), 1124-1131.

Song, Q., \& Jamalipour, A. (2005). Network Selection in an integrated Wireless LAN and UMTS Environment using Mathematical Modeling and Computing Techniques. IEEE Wireless Communications, 12(3), 42-48. https://doi.org/10.1109/MWC.2005.1452853

Stoyanova, M., \& Mahonen, P. (2007). Algorithmic approaches for vertical handoff in Heterogeneous wireless environment. In the Proceedings of IEEE Wireless Communications and Networking Conference, 3780-3785. https://doi.org/10.1109/WCNC.2007.692

Syuhada, M. Z. A., \& Mahamod, I. (2008). Performance Evaluation of Vertical Handoff in Fourth Generation (4G) Networks Model, In the Proceedings of IEEE $6^{\text {th }}$ National Conference on Telecommunication Technologies and IEEE $2^{\text {nd }}$ Malaysia Conference on Photonics, MNTT-MCP, 392-398.

Tomar, G. S. (2009). New Handoff Initiation Algorithm for Cellular Mobile Communication, IEEE. https://doi.org/10.1109/AMS.2009.11

Verma, A., Llora, X., Goldberg, D. E.,\& Campbell, R. H. (2009). Scaling Genetic Algorithms using Mapreduce. In the Proceedings of $9^{\text {th }}$ Internbational Confetrence on Intelligent Systems Design and Applications, 13-18. https://doi.org/10.1109/ISDA.2009.181

Wang, H. J., Katz, R. H., \& Giese, J. (1999) Policy-enabled handoffs across heterogeneous wireless networks. In Proceedings of IEEE $2^{\text {nd }}$ Workshop on Mobile Computing Systems and Applications, LA, USA, 51-60.

Wei, Z., Brahim, B., \& Kee, C. C. (2006). Adaptive quality of service handoff priority scheme for mobile multimedia networks. IEEE Transactions on Vehicular Technology, 494-505.

Weise, T. (2009). Global Optimization Algorithms:Theory and Application (2nn ed.), Germany: it-weise.de.

Ylianttila, M., Pande, M., Makela, J., \& Mahonen, P. (2001). Optimization scheme for mobile users performing vertical handoffs between IEEE 802.11 and GPRS/EDGE Networks. Proceedings of IEEE Global Telecommunications Conference (GLOBECOM'01, San Antonio, Texas, USA, 3439-3443.

Zhu, F., \& McNair, J. (2004). Vertical Handoffs in Fourth-Generation Multinetwork Environments. IEEE Journal of Wireless Communications, 11(3), 8-15. https://doi.org/10.1109/MWC.2004.1308935

\section{Copyrights}

Copyright for this article is retained by the author(s), with first publication rights granted to the journal.

This is an open-access article distributed under the terms and conditions of the Creative Commons Attribution license which permits unrestricted use, distribution, and reproduction in any medium, provided the original work is properly cited. 